\title{
A Survey on Demodex Infections for Undergraduate Students in Some Universities in Ningbo City of Zhejiang Province of China
}

Tonghe Pan, Xianqun Rao, Qi Liao, Zhongjie Lin, Xinyi Zhang, Shengqian Zhu, Xinqian Gu, Xiaobo Cai, Guofeng Chan, Shuangyan Li, Jianfa Liu*

Medical School of Ningbo University, Ningbo, China

Email: "liujianfa@nbu.edu.cn

Received 15 February 2015; accepted 1 March 2015; published 5 March 2015

Copyright (C) 2015 by authors and OALib.

This work is licensed under the Creative Commons Attribution International License (CC BY).

http://creativecommons.org/licenses/by/4.0/

(c) (i) Open Access

\begin{abstract}
Objective: The purpose of this survey is to investigate the university students' face infections with Demodex, and explore the possible factors of Demodex infections. Methods: On this survey, the following pressure method is adopted. The operator has used sterilized thumb against the client part of the facial skin, observing mites under microscopy. Results: The results of the survey for the total Demodex infection rate were $32.50 \%$. Demodex folliculorum infection rate was 39.49\%, Demodex brevis infection rate was $49.74 \%$, and the mixed infection rate was $10.77 \%$. Conclusions: This survey shows that college students' Demodex infection rate is serious according to the corresponding social customs.
\end{abstract}

Keywords

College Students, Demodex Infection, Survey, Ningbo City

Subject Areas: Infectious Diseases, Respiratory Medicine

\section{Introduction}

Demodex families are kinds of small permanent parasitic mites, namely sebaceous follicle Demodex and brevis Demodex. The two parasites mainly parasitize the body of man's forehead, nose, chin and zygomatic places. Mechanical and chemical stimulation can cause skin diseases such as acne, seborrheic dermatitis, and folliculitis

${ }^{*}$ Corresponding author.

How to cite this paper: Pan, T.H., et al. (2015) A Survey on Demodex Infections for Undergraduate Students in Some Universities in Ningbo City of Zhejiang Province of China. Open Access Library Journal, 2: e1214.

http://dx.doi.org/10.4236/oalib.1101214 
[1]. As a result of investigation, Demodex infection rate presents the different levels. Thus, we had investigated the Demodex infections in College students of Ningbo City from December 2012 to March 2013. The results have been shown as follows.

\section{Materials}

Investigated people: totally 600 people, 336 boys and 264 girls at 2009-2011 grades of college students of Ningbo City.

\section{Methods}

Survey methods [2]: The survey contents include personal diet, accommodation environment, hygiene, etc. At the same time, recording client's age, gender, facial disorders, health situation, the collective life and infection degree standards

Checking out any Demodex infections are considered to be positive. Infection degrees of each sample are calculated, all degrees are divided into 3 levels, level 1 is to be degree I (mild); level 2 is to be degree II (moderate); level 3 is to be degree III (serious).

Types of infections: The infection types are divided into three types: sebum Demodex infection, brevis infection and mixed infections with follicles.

All data are treated with spss 12 .

\section{Results}

\subsection{The Infection Rates of Demodex Mites}

All tables showed include 600 university students, among 195 infected students, male's infection rate was 35.71\%, female's infection rate was $28.41 \%$, infection rate between male and female students with $\mathrm{P}$ values were more than 0.05 , which means no statistical significance (Table 1 ).

\subsection{The Infection Degrees with Demodex Mites}

Among 195 students infected, first-degree infection rate accounted for $72.82 \%$, second-degree infection rate accounted for $15.90 \%$, third-degree infection rate accounted for $11.28 \%$ (see Table 2).

\subsection{Facial Diseases and Mite Infection}

The face demonstrated seborrheic dermatitis, folliculitis of Demodex infection rates were greater than the normal facial skin infected, facial diseases and mite infection's P value was less than 0.01 , there was a statistically significant difference (see Table 3).

Table 1. University students' facial Demodex infection rate.

\begin{tabular}{cccc}
\hline Gender & Tested samples & Infection samples & Infection rate (\%) \\
\hline Male & 336 & 120 & 35.71 \\
Female & 264 & 75 & 28.41 \\
Total & 600 & 195 & 32.50 \\
\hline
\end{tabular}

Table 2. Infection degree of university students with facial Demodex mites.

\begin{tabular}{ccc}
\hline Infection degree & Number & Rate $(\%)$ \\
First-degree & 142 & 72.82 \\
Second-degree & 31 & 15.90 \\
Third-degree & 22 & 11.28 \\
\hline
\end{tabular}


Table 3. The Demodex infections of facial skins.

\begin{tabular}{cccc}
\hline The condition of facial skin & Number of tested people & Number of positive people & Positive rate (\%) \\
\hline Acne & 82 & 45 & 54.88 \\
Seborrheic dermatitis & 32 & 15 & 47.34 \\
Folliculitis or hair follicle expansion & 29 & 12 & 41.38 \\
Normal & 457 & 123 & 26.91 \\
\hline
\end{tabular}

\subsection{Conditions of Demodex Infections among Students Who Use Skin Care Products and Those Who Don't Use}

The number of students who use skin care products is 139 , the positive rate was $30.48 \%$, while the number of those who don't use was 56 , positive rate was $38.89 \%$. The positive rate for whom uses skin care products and those with products not used among students with Demodex infections, their P values are greater than 0.05 , means no statistical significance (Table 4).

\subsection{The Ratio of Different Infections with Demodex}

Among 195 students' Demodex infections, simple follicle Demodex are 97 people, simple sebum Demodex are 77 people, and 21 people with mixed infections (Table 5).

\subsection{Demodex Infections among Urban and Rural Students}

Among 473 students who live in rural areas, 153 people are positive, the infection rate was $32.35 \%$. Among 127 students who live in the city, 42 people were positive, urban the infection rate was $33.07 \%$, where $\mathrm{P}$ values were greater than 0.05 , which had no statistical significance (Table 6).

\subsection{Different Ways of Cleansing Demodex Infections of Students}

Cleansing 133 people with water, which is effective for 57 people, the positive rate was $45.86 \%$; cleansing 49 people using soap, which is effective for 49 people, the positive rate was $26.53 \%$; cleansing 411 people using cleanser, which is effective for 123 people, the positive rate of $29.93 \%$; commonly using soap intergrated cleanser to cleanse 7 people, 2 were effective, the positive rate was $28.57 \%$. Calculated with the chi-square test, $\mathrm{P}$ value was less than 0.05 , with highly statistical significance (Table 7).

\subsection{The Treatment of Facial Demodex Cases Using Various Drugs}

Treating students with $2 \%$ mebendazole mixture , 39 cases were negative, negative rate was $78 \%$; oral metronidazole tablets in patients, 43 were negative, negative rate of $86 \%$; oral tinidazole tablets in patients, 44 were negative, negative rate of $88 \%$; Compatibly treated with metronidazole ointment of pure pink and yellow snake patients, there were 47 negative, negative rate was $94 \%$; Compatibly treated with sulfur ointment and glycerol patients, there were 49 negative, negative rate was $98 \%$; patients treated with cream, 13 people were negative, negative rate was $26 \%$. P values were less than 0.01 , which were statistically significant (Table 8 ).

\section{Discussion}

Demodex infections are distributed worldwide, and the population infection rate abroad is about $27 \%-100 \%$ [3], but the infection rate in China is about 20\% - 70\% [4]. The Demodex infection rate of all college students is greatly different, too. In the investigation into College students in some universities in Ningbo City, the Demodex infection rate is $32.50 \%$, which is in accordance with the domestic normal infection rate. The main causes of the differences in the rate of infections with Demodex are attributed to geographical environment, climatic conditions, the season of checking, the site of checking and inspection methods, etc. In this research, we had investigated into the mites infections at the same population using scraping pressure method and sticking method. The results showed that the former method was better than the latter in the nose and face detection. The detection 
Table 4. The comparison of Demodex mites infection between students who use and who don't.

\begin{tabular}{cccc}
\hline Types & Number of tested people & Number of positive people & Positive rate (\%) \\
\hline Skin care products users & 456 & 139 & $30.48 \%$ \\
No skin care products users & 144 & 56 & $38.89 \%$ \\
Total & 600 & 195 & $32.50 \%$ \\
\hline
\end{tabular}

Table 5. Different infection cases.

\begin{tabular}{ccc}
\hline Infected with Demodex & Number of positive infections & Infection rate \\
\hline Simplex mites & 97 & $16.17 \%$ \\
Simplex sebum Demodex & 77 & $12.83 \%$ \\
Mixed infections & 21 & $3.50 \%$ \\
Total & 195 & $32.50 \%$ \\
\hline
\end{tabular}

Table 6. Demodex infections among urban and rural students compared to the different situations.

\begin{tabular}{cccc}
\hline Types & Surveyed & Positive numbers & Positive rate \\
\hline Rural & 473 & 153 & $32.35 \%$ \\
City & 127 & 42 & $33.07 \%$ \\
Total & 600 & 195 & $32.50 \%$ \\
\hline
\end{tabular}

Table 7. Cleansing Demodex infections on students with different ways compared to the infections' situations.

\begin{tabular}{cccc}
\hline Types & Surveyed & Positive numbers & Positive rate \\
\hline Shimizu & 133 & 57 & $45.86 \%$ \\
Soap & 49 & 13 & $26.53 \%$ \\
Cleanser & 411 & 123 & $29.93 \%$ \\
Soap + cleanser & 7 & 2 & $28.57 \%$ \\
Total & 600 & 195 & $32.50 \%$
\end{tabular}

Table 8. The treatment of demodex infections using different drugs.

\begin{tabular}{|c|c|c|c|}
\hline Drugs & Number & Negative numbers & Negative rate \\
\hline Treated with $2 \%$ mixture of mebendazole & 50 & 39 & $78.00 \%$ \\
\hline Treated with Oral metronidazole tablets & 50 & 43 & $86.00 \%$ \\
\hline Treated with Oral tinidazole tablets & 50 & 44 & $88.00 \%$ \\
\hline Pure powders with metronidazole ointment and yellow snake powders & 50 & 47 & $94.00 \%$ \\
\hline Sulfur ointment and glycerol capsules & 50 & 49 & $98.00 \%$ \\
\hline Cream coated & 50 & 13 & $26.00 \%$ \\
\hline
\end{tabular}

rate is association with the amount of sebum. The more the amount of sebum is, the higher the detection rate is [4]. Demodex mites parasitic in hair follicles and sebaceous glands which mechanically stimulate and secrete some pathological factors can damage the hair follicles and sebaceous glands, leading to hair follicle expansion and epithelial degeneration; at the same time, they are also able to lead to the dermal telangiectasia, hyperplasia, 
and blocking of sebaceous gland secretion. The worms can carry pathogenic microorganisms, when at concurrent bacterial infection, causing the hair follicle cell infiltration, fibrous tissue hyperplasia, performance for the local skin flushing, hyperaemia, inflammation, enlarged pores, and rough surfaces. In this paper, the total Demodex infection rate was $32.50 \%$, the infection rate was $35.71 \%$ for males, and $28.41 \%$ for females. The differences from infection rate of male and female are not statistically significant, but most reports such as Zhang [5] find that men have a higher rate of infection than women, the cause of which still needs further study. We had detected the different types of Demodex infections mainly including Demodex follicurolum, Demodex brevis and mixed infections [6]. In infected persons, infections of Degree I accounted for $72.82 \%$ of the total, Degree II infections accounted for $15.90 \%$, and Degree III infections accounted for $11.28 \%$, which was consistent with the infections of Demodex mites reported before [7]. For patients with or without acne, our survey showed that infection rate of patients with acne was higher than those without acne, which had statistical significance. So, Demodex is one of the causes of acne for patients [8], and water cleansing is the destruction of drying and hightemperature conditions, making people susceptible to infection of vermiform mite. But if using soap and cleanser alkaline substances, normal human can resist the infection of vermiform mite. In the experimental groups with different treatments, the best use of combined sulfur ointment and glycerin coated metronidazole in pure powder and snake yellow ointment to the experimental groups, and the negative rate for infections reached $98 \%$ and $94 \%$. We found that experiments coated with ointment smearing part had important significance for patients, the patients applied in nose and nasal groove parts, and the negative rate was higher than those applied in cheeks. Propagation on Demodex infections of populations who are more vulnerable to infection is necessary. Therefore, to prevent infection, we should enhance propaganda, enable students to understand the knowledge on Demodex prevention, developing good health habits, washing supplies special, paying attention to personal hygiene, keeping the face clean, trying to avoid close contact with patients, and cutting off the transmission route [9]. Patients with being positive for infections with acne, rosacea and other facial diseases should be timely treated, lest loss of illness would be incurred because of delay [10]-[12].

\section{References}

[1] Zhou, S.H. and Wang, L.L. (2006) Review on the Biological Research of Human Demodex. Chinese Journal of Parasitology and Parasitic Diseases, 24, 379-384.

[2] Zhang, J., Wang, W., Fu, H., et al. (2012) Analysis of Demodex Infection among College Students Using Skin Scrapings Collected with Cellophane Tape during the Daytime. Journal of pathogen Biology, 7, 454-456.

[3] Zhao, Y.-E. and Guo, N. (2011) Sociodemographic Characteristics and Risk Factor Analysis of Demodex Infestation (Acari: Demodicidae). Zhejiang Univ-Sci B (Biomed \& Biotechnol), 12, 995-1007.

[4] Zhao, Y.E., Guo, N. and Wu, L.P. (2009) The Effect of Temperature on the Viability of Demodex folliculorum and Demodex brevis. Parasitology Research, 105, 1623-1628. http://dx.doi.org/10.1007/s00436-009-1603-x

[5] Zhang, M.H., Xia, H. and Hu, S.F. (2005) Investigation on Demodex Infection in Undergraduates and Observation on Treatment. J Trop Dis. Parasitol, 3, 214-217.

[6] Ha, L.-N., Yang, F.-Q. and Ha, X.-L. (2012) Analysis of Face Infection of Demodex Mite in Medical College Students between 2000 and 2009. Modern Preventive Medicine, 39, 4795-4798.

[7] Xiong, M.Y., Fu, F.D. and Zhou, Y. (2011) Survey on Demodex Infection in College Students. Medical Research and Education, 28, 41-50.

[8] Qin, Y.-H., Zheng, L.-L., Dai, X.-D., et al. (2013) Infection of Follicle Mite (Demodex Mite) in College Students: Survey and Analysis. Chinese Journal of Microecology, 25, 718-719.

[9] Cao, Y.S., You, Q.X. and Wang, L. (2009) Facial Demodex Infection among College Students in Tangshan. Chinese Journal of Parasitology and Parasitic Diseases, 27, 271-273.

[10] Wang, A.H. and Wang, T. (2008) Analysis of the Relation between the Facial Skin Diseases and the Infection of Demodicid Mites in Teenagers. Modern Preventive Medicine, 35, 3396-3399.

[11] Zhan, X.M. (2001) Human Parasitology. 5th Edition, People's Medical Publishing House, Beijing, 173-175.

[12] Li, Y.L. (2008) Human Parasitology. 7th Edition, People's Medical Publishing House, Beijing, 252-254. 